\title{
Indestructibility and Stationary Reflection ${ }^{* \dagger}$
}

\author{
Arthur W. Apter \\ Department of Mathematics \\ Baruch College of CUNY \\ New York, New York 10010 USA \\ and \\ The CUNY Graduate Center, Mathematics \\ 365 Fifth Avenue \\ New York, New York 10016 USA \\ http://faculty.baruch.cuny.edu/apter \\ awapter@alum.mit.edu \\ January 8, 2008 \\ (revised March 21, 2008)
}

\begin{abstract}
If $\kappa<\lambda$ are such that $\kappa$ is a strong cardinal whose strongness is indestructible under $\kappa$-strategically closed forcing and $\lambda$ is weakly compact, then we show that $A=\{\delta<\kappa \mid \delta$ is a non-weakly compact Mahlo cardinal which reflects stationary sets $\}$ must be unbounded in $\kappa$. This phenomenon, however, need not occur in a universe with relatively few large cardinals. In particular, we show how to construct a model where no cardinal is supercompact up to a Mahlo cardinal in which the least supercompact cardinal $\kappa$ is also the least strongly compact cardinal, $\kappa$ 's strongness is indestructible under $\kappa$-strategically closed forcing, $\kappa$ 's supercompactness is indestructible under $\kappa$-directed closed forcing not adding any new subsets of $\kappa$, and $\delta$ is Mahlo and reflects stationary sets iff $\delta$ is weakly compact. In this model, no strong cardinal $\delta<\kappa$ is indestructible under $\delta$-strategically closed forcing. It therefore follows that it is relatively consistent for the least strong cardinal $\kappa$ whose strongness is indestructible under $\kappa$-strategically closed forcing to be the same as the least supercompact cardinal, which also has its supercompactness indestructible under $\kappa$-directed closed forcing not adding any new subsets of $\kappa$.
\end{abstract}

*2000 Mathematics Subject Classifications: 03E35, 03E55.

${ }^{\dagger}$ Keywords: Supercompact cardinal, strong cardinal, weakly compact cardinal, Mahlo cardinal, indestructibility, stationary reflection, non-reflecting stationary set of ordinals.

${ }^{\ddagger}$ The author’s research was partially supported by PSC-CUNY grants and CUNY Collaborative Incentive grants. 


\section{Introduction and Preliminaries}

It is known that the structure of the universe above either a strong or supercompact cardinal $\kappa$ exhibiting suitable indestructibility properties can affect what happens at large cardinals below $\kappa$, assuming the universe is sufficiently rich. On the other hand, these effects can be mitigated if the universe contains relatively few large cardinals. For instance, as shown in [4] and [1], if $\kappa$ is either strong or supercompact and appropriately indestructible and $\lambda>\kappa$ is $2^{\lambda}$ supercompact, then there must always be measurable cardinals $\delta<\kappa$ which are not themselves limits of measurable cardinals such that $\delta$ is $\delta^{+}$strongly compact but $\delta$ is not $\delta^{+}$supercompact. On the other hand, as shown in [1], the existence of such measurable cardinals below a suitably indestructible strong or supercompact cardinal $\kappa$ need not occur if there are no measurable cardinals above $\kappa$. This and related phenomena have been examined further in [4], [1], and [2].

The purpose of this paper is to continue the studies mentioned in the preceding paragraph, but in the context of stationary reflection. Specifically, we prove the following.

Theorem 1 If $\kappa<\lambda$ are such that $\kappa$ is a strong cardinal whose strongness is indestructible under $\kappa$-strategically closed forcing and $\lambda$ is weakly compact, then $A=\{\delta<\kappa \mid \delta$ is a non-weakly compact Mahlo cardinal which reflects stationary sets\} is unbounded in $\kappa$.

Theorem 2 Suppose $V \vDash " Z F C+G C H+\kappa$ is supercompact + No cardinal is supercompact up to a Mahlo cardinal". There is then a partial ordering $\mathbb{P} \in V$ such that $V^{\mathbb{P}} \vDash " Z F C+\kappa$ is supercompact + No cardinal is supercompact up to a Mahlo cardinal". In $V^{\mathbb{P}}, \kappa$ is both the least strongly compact and least supercompact cardinal. In addition, in $V^{\mathbb{P}}, \kappa^{\prime}$ s strongness is indestructible under $\kappa$-strategically closed forcing, and $\kappa$ 's supercompactness is indestructible under $\kappa$-directed closed forcing not adding any new subsets of $\kappa$. Finally, in $V^{\mathbb{P}}, \delta$ is Mahlo and reflects stationary sets iff $\delta$ is weakly compact.

As a corollary to Theorem 2, we have the following theorem.

Theorem 3 It is consistent, relative to the existence of a supercompact cardinal, for the least strong cardinal $\kappa$ whose strongness is indestructible under $\kappa$-strategically closed forcing to be the 
same as the least supercompact cardinal, which also has its supercompactness indestructible under $\kappa$-directed closed forcing not adding any new subsets of $\kappa$.

Theorem 3 should be contrasted with [3, Lemma 2.1], which says that any supercompact cardinal must be a limit of strong cardinals. It is thus not possible for the least supercompact cardinal to be the same as the least strong cardinal. If we add on the requirement of a certain degree of indestructibility, then Theorem 3 indicates that not only can the least strong cardinal with this degree of indestructibility be the same as the least supercompact cardinal $\kappa$, but the supercompactness of $\kappa$ can have a certain amount of indestructibility as well.

We now very briefly give some preliminary information concerning notation and terminology. When forcing, $q \geq p$ means that $q$ is stronger than $p$. If $G$ is $V$-generic over $\mathbb{P}$, we will abuse notation slightly and use both $V[G]$ and $V^{\mathbb{P}}$ to indicate the universe obtained by forcing with $\mathbb{P}$. We will, from time to time, confuse terms with the sets they denote and write $x$ when we actually mean $\dot{x}$ or $\check{x}$.

The partial ordering $\mathbb{P}$ is $\kappa$-directed closed if every directed set of conditions of size less than $\kappa$ has an upper bound. $\mathbb{P}$ is $\kappa$-strategically closed if in the two person game in which the players construct an increasing sequence $\left\langle p_{\alpha} \mid \alpha \leq \kappa\right\rangle$, where player I plays odd stages and player II plays even and limit stages (choosing the trivial condition at stage 0), player II has a strategy which ensures the game can always be continued. $\mathbb{P}$ is $\prec \kappa$-strategically closed if in the two person game in which the players construct an increasing sequence $\left\langle p_{\alpha} \mid \alpha<\kappa\right\rangle$, where player I plays odd stages and player II plays even and limit stages (choosing the trivial condition at stage 0), player II has a strategy which ensures the game can always be continued. $\mathbb{P}$ is $<\kappa$-strategically closed if $\mathbb{P}$ is $\delta$-strategically closed for all cardinals $\delta<\kappa . \mathbb{P}$ is $(\kappa, \infty)$-distributive if given a sequence $\left\langle D_{\alpha} \mid \alpha<\kappa\right\rangle$ of dense open subsets of $\mathbb{P}, \bigcap_{\alpha<\kappa} D_{\alpha}$ is also a dense open subset of $\mathbb{P}$. Note that if $\mathbb{P}$ is either $\kappa$-strategically closed or $(\kappa, \infty)$-distributive and $f: \kappa \rightarrow V$ is a function in $V^{\mathbb{P}}$, then $f \in V$. In addition, if $\mathbb{P}$ is $\kappa$-directed closed, then $\mathbb{P}$ is $\prec \kappa$-strategically closed. The cardinal $\kappa$ reflects stationary sets if for every stationary $S \subseteq \kappa$, there is some $\delta<\kappa$ such that $S \cap \delta$ is stationary in $\delta$. The cardinal $\kappa$ is supercompact up to the cardinal $\lambda$ if $\kappa$ is $\delta$ supercompact for every $\delta<\lambda$. 
As in [8], if $\mathcal{A}$ is a collection of partial orderings, then the lottery sum is the partial ordering $\oplus \mathcal{A}=\{\langle\mathbb{P}, p\rangle \mid \mathbb{P} \in \mathcal{A}$ and $p \in \mathbb{P}\} \bigcup\{0\}$, ordered with 0 below everything and $\langle\mathbb{P}, p\rangle \leq\left\langle\mathbb{P}^{\prime}, p^{\prime}\right\rangle$ iff $\mathbb{P}=\mathbb{P}^{\prime}$ and $p \leq p^{\prime}$. Intuitively, if $G$ is $V$-generic over $\oplus \mathcal{A}$, then $G$ first selects an element of $\mathcal{A}$ (or as Hamkins says in [8], "holds a lottery among the posets in $\mathcal{A}$ ") and then forces with it. The terminology "lottery sum" is due to Hamkins, although the concept of the lottery sum of partial orderings has been around for quite some time and has been referred to at different junctures via the names "disjoint sum of partial orderings," "side-by-side forcing," and "choosing which partial ordering to force with generically."

\section{The Proofs of Theorems $1-3$}

We turn now to the proof of Theorem 1, which we begin with the following key lemma.

Lemma 2.1 Suppose $V \vDash " \kappa<\lambda$ are such that $\kappa$ is a regular cardinal and $\lambda$ is weakly compact". There is then a $\kappa$-strategically closed notion of forcing $\mathbb{Q} \in V$ such that $V^{\mathbb{Q}} \vDash$ " $\lambda$ is a non-weakly compact Mahlo cardinal which reflects stationary sets".

Proof: Let $\mathbb{P}$ be the reverse Easton iteration of length $\lambda+1$ which adds a Cohen subset to every inaccessible cardinal in the half-open interval $(\kappa, \lambda]$. We begin by showing that $V^{\mathbb{P}} \vDash$ " $\lambda$ is weakly compact".

To do this, we adapt an argument from [8, Theorem 1.4], quoting liberally from Hamkins' presentation. Write $\mathbb{P}=\mathbb{P}_{\lambda} * \dot{\mathbb{Q}}_{\lambda}$. By the definition of $\mathbb{P}$, it is clearly the case that $\lambda$ remains inaccessible in $V^{\mathbb{P}}=V^{\mathbb{P}_{\lambda} * \dot{\mathbb{Q}}_{\lambda}}$. It therefore is enough to show that $\lambda$ has the tree property in $V^{\mathbb{P}_{\lambda} * \dot{\mathbb{Q}}_{\lambda}}$. Suppose as a consequence that $\dot{T}$ is a name for a $\lambda$-tree in $V^{\mathbb{P}_{\lambda} * \dot{\mathbb{Q}}_{\lambda}}$. In $V$, let $N$ be a transitive elementary substructure of $H\left(\lambda^{+}\right)$of size $\lambda$ containing $\mathbb{P}_{\lambda} * \dot{\mathbb{Q}}_{\lambda}$ and $\dot{T}$ which is closed under $<\lambda$ sequences. Since $\lambda$ is weakly compact in $V$, there is an elementary embedding $j: N \rightarrow M$ having critical point $\lambda$. As in [8, Theorem 1.4], we may also assume that $|M|=\lambda$ and $V \vDash " M^{<\lambda} \subseteq M^{\prime}$.

Write $j\left(\mathbb{P}_{\lambda}\right)=\mathbb{P}_{\lambda} * \dot{\mathbb{Q}}_{\lambda} * \dot{\mathbb{R}} * j\left(\dot{\mathbb{Q}}_{\lambda}\right)$. For any $V$-generic object $G * H$ over $\mathbb{P}_{\lambda} * \dot{\mathbb{Q}}_{\lambda}$, the fact $\mathbb{P}_{\lambda}$ is $\lambda$-c.c. and $\mathbb{Q}_{\lambda}$ is $\lambda$-directed closed allows us to infer that $V[G][H] \vDash " M[G][H]^{<\lambda} \subseteq M[G][H] "$. Further, it is the case that $M[G][H] \vDash " \mathbb{R} * j\left(\dot{\mathbb{Q}}_{\lambda}\right)$ is $\prec \lambda$-strategically closed", since $M[G][H] \vDash " \mathbb{R} * j\left(\dot{\mathbb{Q}}_{\lambda}\right)$ is 
$\lambda$-directed closed". From this, because $V[G][H] \vDash " M[G][H]^{<\lambda} \subseteq M[G][H] ", V[G][H] \vDash " \mathbb{R} * j\left(\dot{\mathbb{Q}}_{\lambda}\right)$

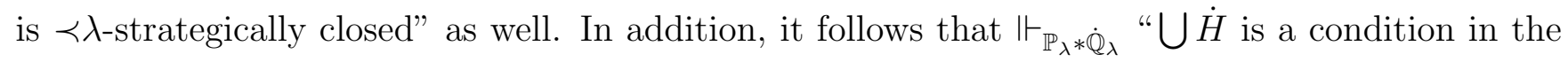
partial ordering $j\left(\dot{\mathbb{Q}}_{\lambda}\right) \in N^{\mathbb{P}_{\lambda} * \dot{\mathbb{Q}}_{\lambda} * \dot{\mathbb{R}}}$ ". Therefore, since by the fact $V[G][H] \vDash "|M[G][H]|=\lambda "$, there are only $\lambda$ many dense open subsets of $\mathbb{R} * j\left(\dot{\mathbb{Q}}_{\lambda}\right)$ present in $M[G][H]$, we may use a standard diagonalization argument to meet the $\lambda$ many dense open subsets of $\mathbb{R} * j\left(\dot{\mathbb{Q}}_{\lambda}\right)$ and construct in $V[G][H]$ an $M[G][H]$-generic object $H^{\prime} * H^{\prime \prime}$ for $\mathbb{R} * j\left(\dot{\mathbb{Q}}_{\lambda}\right)$ such that $\bigcup H \in H^{\prime \prime}$. Specifically, let $\left\langle D_{\sigma} \mid \sigma<\lambda\right\rangle$ enumerate in $V[G][H]$ the dense open subsets of $\mathbb{R} * j\left(\dot{\mathbb{Q}}_{\lambda}\right)$ present in $M[G][H]$ such that every dense open subset of $\mathbb{R} * j\left(\dot{\mathbb{Q}}_{\lambda}\right)$ occurring in $M[G][H]$ appears at an odd stage at least once in the enumeration. If $\sigma$ is an odd ordinal, $\sigma=\tau+1$ for some $\tau$. Player I picks $p_{\sigma} \in D_{\sigma}$ extending $q_{\tau}$ (initially, $q_{0}=\langle\emptyset, \bigcup H\rangle$ ), and player II responds by picking $q_{\sigma} \geq p_{\sigma}$ according to a fixed strategy $\mathcal{S}$ (so $q_{\sigma} \in D_{\sigma}$ ). If $\sigma$ is a limit ordinal, player II uses $\mathcal{S}$ to pick $q_{\sigma}$ extending each $q \in\left\langle q_{\beta} \mid \beta<\sigma\right\rangle$. By the $\prec \lambda$-strategic closure of $\mathbb{R} * j\left(\dot{\mathbb{Q}}_{\lambda}\right)$ in $V[G][H]$, player II's strategy can be assumed to be a winning one, so $\left\langle q_{\sigma} \mid \sigma<\lambda\right\rangle$ can be taken as an increasing sequence of conditions with $q_{\sigma} \in D_{\sigma}$ for $\sigma<\lambda$. Let $H^{\prime}=\left\{p \in \mathbb{R} * j\left(\dot{\mathbb{Q}}_{\lambda}\right) \mid \exists \sigma<\lambda\left[q_{\sigma} \geq p\right]\right\}$. As $j^{\prime \prime}(G * H) \subseteq G * H * H^{\prime} * H^{\prime \prime}$, we then have that $j$ lifts in $V[G][H]$ to an elementary embedding $j: N[G][H] \rightarrow M[G][H]\left[H^{\prime}\right]\left[H^{\prime \prime}\right]$.

Because $\dot{T} \in N, T \in N[G][H]$. Since $T$ is a $\lambda$-tree in both $V[G][H]$ and $N[G][H]$, by elementarity, $j(T)$ is a $j(\lambda)$-tree in $M[G][H]\left[H^{\prime}\right]\left[H^{\prime \prime}\right]$. Any element on the $\lambda^{\text {th }}$ level of of $j(T)$ gives a branch of length $\lambda$ through $T$. This means that $\lambda$ has the tree property in $V[G][H]$, as desired.

We continue using results of Kunen found in the proof of the Theorem of [9, Section 3, pages 68-71]. Working now in $V^{\mathbb{P}_{\lambda}}$, we may factor $\mathbb{Q}_{\lambda}$ as $\mathbb{R}_{\lambda} * \dot{\mathbb{T}}_{\lambda}$, where $\mathbb{R}_{\lambda}$ is a notion of forcing adding a $\lambda$-Souslin tree $\mathcal{T}$, and $\mathbb{T}_{\lambda}$ is the partial ordering adding a generic path through $\mathcal{T}$. It is the case (see $\left[9\right.$, pages 69-70]) that $\mathbb{R}_{\lambda}$ is $<\lambda$-strategically closed and $\mathbb{T}_{\lambda}$ is $\lambda$-c.c. In addition, $\mathbb{R}_{\lambda}$ is $\aleph_{1}$-directed closed but not necessarily $\aleph_{2}$-directed closed. We claim that $\mathbb{Q}=\mathbb{P}_{\lambda} * \dot{\mathbb{R}}_{\lambda}$ is our desired partial ordering.

To see this, by its definition, $\mathbb{Q}$ is clearly $\kappa$-strategically closed. Since $V^{\mathbb{P}_{\lambda} * \dot{\mathbb{Q}}_{\lambda}} \vDash$ " $\lambda$ is weakly compact", $V^{\mathbb{P}_{\lambda} * \dot{\mathbb{Q}}_{\lambda}} \vDash$ " $\lambda$ is a Mahlo cardinal". Thus, since $\mathbb{Q}_{\lambda}=\mathbb{R}_{\lambda} * \dot{\mathbb{T}}_{\lambda}$, forcing can't create a Mahlo cardinal, and $V^{\mathbb{P}_{\lambda} * \dot{\mathbb{R}}_{\lambda}} \vDash$ "There is a $\lambda$-Souslin tree", $V^{\mathbb{P}_{\lambda} * \mathbb{R}_{\lambda}} \vDash$ " $\lambda$ is a non-weakly compact 
Mahlo cardinal". It therefore remains to show that $V^{\mathbb{P}_{\lambda} * \mathbb{\mathbb { R }}_{\lambda}} \vDash$ " $\lambda$ reflects stationary sets". To do this, let $S \in V^{\mathbb{P}_{\lambda} * \dot{\mathbb{R}}_{\lambda}}$ be stationary. Since $\mathbb{T}_{\lambda}$ is $\lambda$-c.c., by [10, Exercise H2, page 247], $V^{\mathbb{P}_{\lambda} * \dot{\mathbb{R}}_{\lambda} * \dot{\mathbb{T}}_{\lambda}}=$ $V^{\mathbb{P}_{\lambda} * \dot{\mathbb{Q}}_{\lambda}} \vDash$ "S is stationary". Another appeal to the weak compactness of $\lambda$ in $V^{\mathbb{P}_{\lambda} * \dot{\mathbb{Q}}_{\lambda}}$ then yields the existence of a fixed $\delta<\lambda$ such that $V^{\mathbb{P}_{\lambda} * \dot{\mathbb{Q}}_{\lambda}} \vDash " S \cap \delta$ is a stationary subset of $\delta$ ". Because $V^{\mathbb{P}_{\lambda}} \vDash " \mathbb{Q}_{\lambda}$ is $\lambda$-directed closed", $S \cap \delta \in V^{\mathbb{P}_{\lambda}}$, and $V^{\mathbb{P}_{\lambda}} \vDash " S \cap \delta$ is a stationary subset of $\delta$ ". Since $\mathbb{R}_{\lambda}$ is $\left\langle\lambda\right.$-strategically closed, $V^{\mathbb{P}_{\lambda} * \dot{\mathbb{R}}_{\lambda}}=V^{\mathbb{Q}} \vDash " S \cap \delta$ is a stationary subset of $\delta$ ". This completes the proof of Lemma 2.1 .

We note that the proof given in Lemma 2.1 of the fact that $V^{\mathbb{P}_{\lambda} * \dot{\mathbb{Q}}_{\lambda}} \vDash$ " $\lambda$ is weakly compact" remains valid if we only force with $\mathbb{P}_{\lambda}$. In this case, $N$ may be chosen so as to contain $\mathbb{P}_{\lambda}$ as a member, with $j: N \rightarrow M$ as before an elementary embedding having critical point $\lambda$. We then have that $j\left(\mathbb{P}_{\lambda}\right)=\mathbb{P}_{\lambda} * \dot{\mathbb{R}}^{*}$, and there is neither a forcing nor a generic object $H$ at stage $\lambda$ in $V[G]$ (although in $M[G], \mathbb{R}^{*}$ contains a forcing at stage $\lambda$ ). Since $M[G] \vDash$ " $\mathbb{R}^{*}$ is $\prec \lambda$-strategically closed", an $M[G]$-generic object $H^{*}$ for $\mathbb{R}^{*}$ may be constructed in $V[G]$ as above, without having to worry about a master condition for a forcing done at stage $\lambda$ in $V[G]$. (The game starts with $q_{0}$ being the empty condition.) As $j^{\prime \prime} G \subseteq G * H^{*}$, the elementary embedding $j$ will then lift to $j: N[G] \rightarrow M[G]\left[H^{*}\right]$, and the remainder of the argument that $V[G] \vDash$ " $\lambda$ is weakly compact" is as before. This observation will be critical in the proof of Lemma 2.6 to be given in the proof of Theorem 2 .

Having completed the proof of Lemma 2.1, the proof of Theorem 1 now follows fairly easily. Suppose $\kappa<\lambda$ are such that $\kappa$ is a strong cardinal whose strongness is indestructible under $\kappa$ strategically closed forcing and $\lambda$ is weakly compact. Force with the $\kappa$-strategically closed partial ordering $\mathbb{Q}$ of Lemma 2.1. After this forcing, $\lambda$ has become a non-weakly compact Mahlo cardinal which reflects stationary sets, and $\kappa$ remains a strong cardinal. In addition, by reflection, $A=$ $\{\delta<\kappa \mid \delta$ is a non-weakly compact Mahlo cardinal which reflects stationary sets $\}$ is unbounded in $\kappa$. Since $\mathbb{Q}$ is $\kappa$-strategically closed, $A$ must be unbounded in $\kappa$ in the ground model as well. This completes the proof of Theorem 1 . 
With the proof of Theorem 1 finished, we turn now to the proof of Theorem 2.

Proof: Let $V \vDash$ "ZFC $+\mathrm{GCH}+\kappa$ is supercompact + No cardinal is supercompact up to a Mahlo cardinal". Our partial ordering $\mathbb{P}$ is the reverse Easton iteration $\left\langle\left\langle\mathbb{P}_{\alpha}, \dot{\mathbb{Q}}_{\alpha}\right\rangle \mid \alpha<\kappa\right\rangle$ of length $\kappa$ which begins by adding a Cohen real and whose only later nontrivial stages occur at ordinals $\delta<\kappa$ which are $V$-Mahlo cardinals. If $\delta$ is not weakly compact in $V$, then $\dot{\mathbb{Q}}_{\delta}$ is a term for the partial ordering adding a non-reflecting stationary set of ordinals of cofinality $\omega$ to $\delta .^{1}$ If $\delta$ is weakly compact in $V$, then $\dot{\mathbb{Q}}_{\delta}$ is a term for the lottery sum of all partial orderings having rank below the least $V$-Mahlo cardinal above $\delta$ which are either $\delta$-strategically closed or $\delta$-directed closed and do not add a new subset of $\delta$.

Lemma 2.2 $V^{\mathbb{P}} \vDash$ "No cardinal is supercompact up to a Mahlo cardinal".

Proof: Write $\mathbb{P}=\mathbb{P}^{\prime} * \dot{\mathbb{P}}^{\prime \prime}$, where $\mathbb{P}^{\prime}$ is nontrivial, $\left|\mathbb{P}^{\prime}\right|=\omega$, and $\Vdash_{\mathbb{P}^{\prime}}$ " $\dot{\mathbb{P}}^{\prime \prime}$ is $\aleph_{1}$-strategically closed". Because $\mathbb{P}$ admits this factorization, by Hamkins' Gap Forcing Theorem of [6] and [7], if $V^{\mathbb{P}} \vDash$ " $\delta$ is $\lambda$ supercompact", then $V \vDash$ " $\delta$ is $\lambda$ supercompact" as well. Thus, since $V \vDash$ "No cardinal is supercompact up to a Mahlo cardinal", $V^{\mathbb{P}} \vDash$ "No cardinal is supercompact up to a Mahlo cardinal". This completes the proof of Lemma 2.2 .

Lemma 2.3 $V^{\mathbb{P}} \vDash$ “ $\kappa$ 's supercompactness is indestructible under $\kappa$-directed closed forcing not adding any new subsets of $\kappa "$.

Proof: We follow the proof of $\left[1\right.$, Lemma 1.1]. Let $\mathbb{Q} \in V^{\mathbb{P}}$ be such that $V^{\mathbb{P}} \vDash " \mathbb{Q}$ is $\kappa$-directed closed and does not add a new subset of $\kappa$ ". Take $\dot{\mathbb{Q}}$ as a term for $\mathbb{Q}$ such that $\Vdash_{\mathbb{P}}$ " $\dot{\mathbb{Q}}$ is $\kappa$-directed closed and does not add a new subset of $\kappa^{\prime \prime}$. Suppose $\lambda \geq \max \left(\kappa^{+},|\mathrm{TC}(\dot{\mathbb{Q}})|\right)$ is an arbitrary cardinal, and let $\gamma=2^{\mid[\lambda]^{<\kappa \mid}}$. Take $j: V \rightarrow M$ as an elementary embedding witnessing the $\gamma$

\footnotetext{
${ }^{1}$ The precise definition of this partial ordering may be found in [3]. We do wish to note here, however, that it is $\prec \delta$-strategically closed. In particular, the partial ordering for adding a non-reflecting stationary set of ordinals of cofinality $\omega$ to $\delta^{+}$is $\delta$-strategically closed.
} 
supercompactness of $\kappa$ generated by a supercompact ultrafilter over $P_{\kappa}(\gamma)$. Since $V \vDash$ "No cardinal above $\kappa$ is Mahlo", $\gamma \geq \kappa$, and $M^{\gamma} \subseteq M, M \vDash$ " $\kappa$ is Mahlo and no cardinal in the half-open interval $(\kappa, \gamma]$ is Mahlo". In addition, by our assumptions, $\mathbb{Q}$ is an allowable choice in the stage $\kappa$ lottery held in $M^{\mathbb{P}}$ in the definition of $j(\mathbb{P})$. Hence, by forcing above a condition opting for $\mathbb{Q}, j(\mathbb{P} * \dot{\mathbb{Q}})$ is forcing equivalent to $\mathbb{P} * \dot{\mathbb{Q}} * \dot{\mathbb{R}} * j(\dot{\mathbb{Q}})$, where the first nontrivial stage of forcing in $\dot{\mathbb{R}}$ takes place well above $\gamma$. Laver's original argument from [11] now applies and shows $V^{\mathbb{P} * \dot{\mathbb{Q}}} \vDash$ " $\kappa$ is $\lambda$ supercompact". (Simply let $G_{0} * G_{1} * G_{2}$ be $V$-generic over $\mathbb{P} * \dot{\mathbb{Q}} * \dot{\mathbb{R}}$, lift $j$ in $V\left[G_{0}\right]\left[G_{1}\right]\left[G_{2}\right]$ to $j: V\left[G_{0}\right] \rightarrow M\left[G_{0}\right]\left[G_{1}\right]\left[G_{2}\right]$, take a master condition $p$ for $j^{\prime \prime} G_{1}$ and a $V\left[G_{0}\right]\left[G_{1}\right]\left[G_{2}\right]$-generic object $G_{3}$ over $j(\mathbb{Q})$ containing $p$, lift $j$ again in $V\left[G_{0}\right]\left[G_{1}\right]\left[G_{2}\right]\left[G_{3}\right]$ to $j: V\left[G_{0}\right]\left[G_{1}\right] \rightarrow M\left[G_{0}\right]\left[G_{1}\right]\left[G_{2}\right]\left[G_{3}\right]$, and show by the $\gamma^{+}-$ directed closure of $\mathbb{R} * j(\dot{\mathbb{Q}})$ that the supercompactness measure over $\left(P_{\kappa}(\lambda)\right)^{V\left[G_{0}\right]\left[G_{1}\right]}$ generated by $j$ is actually a member of $V\left[G_{0}\right]\left[G_{1}\right]$.) As $\lambda$ and $\mathbb{Q}$ were arbitrary, this completes the proof of Lemma 2.3 .

Since trivial forcing is $\kappa$-directed closed and does not add any new subsets of $\kappa$, Lemma 2.3 immediately allows us to infer that $V^{\mathbb{P}} \vDash$ " $\kappa$ is supercompact".

Lemma 2.4 $V^{\mathbb{P}} \vDash$ “ $\kappa$ 's strongness is indestructible under $\kappa$-strategically closed forcing".

Proof: Let $\mathbb{Q} \in V^{\mathbb{P}}$ be such that $V^{\mathbb{P}} \vDash " \mathbb{Q}$ is $\kappa$-strategically closed". Take $\dot{\mathbb{Q}}$ as a term for $\mathbb{Q}$ such that $\vdash_{\mathbb{P}}$ "Ф் is $\kappa$-strategically closed". Suppose $\lambda \geq \max \left(\kappa^{+},|\mathrm{TC}(\dot{\mathbb{Q}})|\right)$ is an arbitrary cardinal, and let $\gamma$ be the least singular strong limit cardinal of cofinality $\kappa^{+}$above $\lambda$. Take $j: V \rightarrow M$ as an elementary embedding witnessing the $\gamma$ strongness of $\kappa$ generated by a $(\kappa, \gamma)$-extender such that $M=\left\{j(f)(a) \mid a \in[\gamma]^{<\omega}, f \in V\right.$, and $\left.\operatorname{dom}(f)=[\kappa]^{|a|}\right\}$. Since $V \vDash$ "No cardinal above $\kappa$ is Mahlo" and $V_{\gamma} \subseteq M$, by the choice of $\gamma, M \vDash " \kappa$ is Mahlo and no cardinal in the half-open interval $(\kappa, \gamma]$ is Mahlo". In addition, as in Lemma 2.3, by our assumptions, $\mathbb{Q}$ is an allowable choice in the stage $\kappa$ lottery held in $M^{\mathbb{P}}$ in the definition of $j(\mathbb{P})$. Hence, by forcing above a condition $p$ opting for $\mathbb{Q}$, we may assume that $j(\mathbb{P} * \dot{\mathbb{Q}})$ is forcing equivalent to $\mathbb{P} * \dot{\mathbb{Q}} * \dot{\mathbb{R}} * j(\dot{\mathbb{Q}})$, where the first nontrivial stage of forcing in $\dot{\mathbb{R}}$ takes place well above $\gamma$. Consequently, to complete the proof of Lemma 2.4, we show that in $V^{\mathbb{P} * \dot{\mathbb{Q}}}, j$ lifts to a $\gamma$ strongness embedding $j: V^{\mathbb{P} * \dot{\mathbb{Q}}} \rightarrow M^{j(\mathbb{P} * \dot{\mathbb{Q}})}$. 
To do this, we use ideas found in the proof of [8, Theorem 4.10]. (See also [5, Lemma 2.2].) For the benefit of readers, we give the argument here as well, once again taking the liberty to quote freely from Hamkins' presentation. Since by choice of $\gamma$, we may also assume that $M^{\kappa} \subseteq M$, this means that if $G$ is $V$-generic over $\mathbb{P}$ and $H$ is $V[G]$-generic over $\mathbb{Q}, \mathbb{R}$ is $\prec \kappa^{+}$-strategically closed in both $V[G][H]$ and $M[G][H]$, and $\mathbb{R}$ is $\gamma$-strategically closed in $M[G][H]$.

As in [8], by using a suitable coding which allows us to identify finite subsets of $\gamma$ with elements of $\gamma$, the definition of $M$ enables us to find some $\alpha<\gamma$ and function $g$ such that $\dot{\mathbb{Q}}=j(g)(\alpha)$. Let $N=\left\{\operatorname{den}_{G * H}(\dot{z}) \mid \dot{z}=j(f)(\kappa, \alpha, \gamma)\right.$ for some function $\left.f \in V\right\}$. It is easy to verify that $N \prec M[G][H]$, that $N$ is closed under $\kappa$ sequences in $V[G][H]$, and that $\kappa, \alpha, \gamma, p, \mathbb{Q}$, and $\mathbb{R}$ are all elements of $N$. Further, since $\mathbb{R}$ is $j(\kappa)$-c.c. in $M[G][H]$ and there are only $2^{\kappa}=\kappa^{+}$many functions $f: \kappa \rightarrow V_{\kappa}$ in $V$, there are at most $\kappa^{+}$many dense open subsets of $\mathbb{R}$ in $N$. Therefore, since $\mathbb{R}$ is $\prec \kappa^{+}$-strategically closed in both $M[G][H]$ and $V[G][H]$, we can build an $N$-generic object $H^{\prime}$ over $\mathbb{R}$ in $V[G][H]$ using the diagonalization argument given in the proof of Lemma 2.1 (where once again the game starts with $q_{0}$ being the empty condition).

We show now that $H^{\prime}$ is actually $M[G][H]$-generic over $\mathbb{R}$. If $D$ is a dense open subset of $\mathbb{R}$ in $M[G][H]$, then $D=\operatorname{den}_{G * H}(\dot{D})$ for some name $\dot{D} \in M$. Consequently, $\dot{D}=j(f)\left(\kappa, \kappa_{1}, \ldots, \kappa_{n}\right)$ for some function $f \in V$ and $\kappa<\kappa_{1}<\cdots<\kappa_{n}<\gamma$. Let $\bar{D}$ be a name for the intersection of all $\operatorname{den}_{G * H}\left(j(f)\left(\kappa, \alpha_{1}, \ldots, \alpha_{n}\right)\right)$, where $\kappa<\alpha_{1}<\cdots<\alpha_{n}<\gamma$ is such that $j(f)\left(\kappa, \alpha_{1}, \ldots, \alpha_{n}\right)$ yields a name for a dense open subset of $\mathbb{R}$. Since this name can be given in $M$ and $\mathbb{R}$ is $\gamma$-strategically closed in $M[G][H]$ and therefore $(\gamma, \infty)$-distributive in $M[G][H], \bar{D}$ is a name for a dense open subset of $\mathbb{R}$ which is definable without the parameters $\kappa_{1}, \ldots, \kappa_{n}$. Hence, by its definition, $\operatorname{den}_{G * H}(\bar{D}) \in N$. Thus, since $H^{\prime}$ meets every dense open subset of $\mathbb{R}$ present in $N, H^{\prime} \cap \operatorname{den}_{G * H}(\bar{D}) \neq \emptyset$, so since $\bar{D}$ is forced to be a subset of $\dot{D}, H^{\prime} \cap \operatorname{den}_{G * H}(\dot{D}) \neq \emptyset$. This means $H^{\prime}$ is $M[G][H]$-generic over $\mathbb{R}$, so in $V[G][H], j$ lifts to $j: V[G] \rightarrow M[G][H]\left[H^{\prime}\right]$.

It remains to lift $j$ through the forcing $\mathbb{Q}$ while working in $V[G][H]$. To do this, it suffices to show that $j^{\prime \prime} H \subseteq j(\mathbb{Q})$ generates an $M[G][H]\left[H^{\prime}\right]$-generic object $H^{\prime \prime}$ over $j(\mathbb{Q})$. Given a dense open subset $D \subseteq j(\mathbb{Q}), D \in M[G][H]\left[H^{\prime}\right], D=\operatorname{den}_{G * H * H^{\prime}}(\dot{D})$ for some name $\dot{D}=j(\vec{D})(a)$, where 
$a \in[\gamma]^{<\omega}$ and $\vec{D}=\left\langle D_{\sigma} \mid \sigma \in[\kappa]^{|a|}\right\rangle$ is a function. We may assume that every $D_{\sigma}$ is a dense open subset of $\mathbb{Q}$. Since $\mathbb{Q}$ is $(\kappa, \infty)$-distributive, it follows that $D^{\prime}=\bigcap_{\sigma \in[\kappa]^{|a|}} D_{\sigma}$ is also a dense open subset of $\mathbb{Q}$. As $j\left(D^{\prime}\right) \subseteq D$ and $H \cap D^{\prime} \neq \emptyset, j^{\prime \prime} H \cap D \neq \emptyset$. Thus, $H^{\prime \prime}=\left\{p \in j(\mathbb{Q}) \mid \exists q \in j^{\prime \prime} H[q \geq p]\right\}$ is our desired generic object, and $j$ lifts to $j: V[G][H] \rightarrow M[G][H]\left[H^{\prime}\right]\left[H^{\prime \prime}\right]$. This final lifted version of $j$ is $\gamma$ strong since $V_{\gamma} \subseteq M$, meaning $\left(V_{\gamma}\right)^{V[G][H]} \subseteq M[G][H] \subseteq M[G][H]\left[H^{\prime}\right]\left[H^{\prime \prime}\right]$. Therefore, $V[G][H] \vDash$ " $\kappa$ is $\gamma$ strong". As $\lambda$ and $\mathbb{Q}$ were arbitrary, this completes the proof of Lemma 2.4.

Lemma 2.5 $V^{\mathbb{P}} \vDash " \kappa$ is both the least strongly compact and least supercompact cardinal".

Proof: Let $\mathbb{Q} \in V^{\mathbb{P}}$ be the partial ordering for adding a non-reflecting stationary set of ordinals of cofinality $\omega$ to $\kappa^{+}$. Since $V^{\mathbb{P}} \vDash " \mathbb{Q}$ is $\kappa$-strategically closed", by Lemma $2.4, V^{\mathbb{P} * \dot{\mathbb{Q}}} \vDash$ " $\kappa$ is a strong cardinal". Since $V^{\mathbb{P} * \dot{\mathbb{Q}}} \vDash$ " $\kappa^{+}$contains a non-reflecting stationary set of ordinals of cofinality $\omega$ ", by reflection, $V^{\mathbb{P} * \dot{\mathbb{Q}}} \vDash$ "There are unboundedly in $\kappa$ many cardinals $\delta<\kappa$ which contain non-reflecting

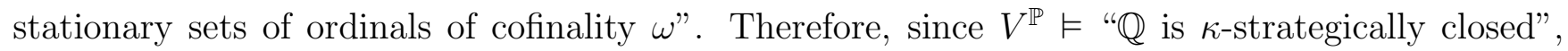
this last fact must be true in $V^{\mathbb{P}}$ as well. By a theorem of Solovay (see [12, Theorem 4.8] and the succeeding remarks), $V^{\mathbb{P}} \vDash$ "No cardinal $\delta<\kappa$ is strongly compact". As $V^{\mathbb{P}} \vDash$ " $\kappa$ is supercompact", $V^{\mathbb{P}} \vDash " \kappa$ is both the least strongly compact and least supercompact cardinal". This completes the proof of Lemma 2.5.

Lemma 2.6 Suppose that $\delta<\kappa$ is weakly compact. Then $V^{\mathbb{P}_{\delta}} \vDash " \delta$ is weakly compact".

Proof: In analogy to the proof of Lemma 2.1, let $\dot{T}$ be the name of a $\delta$-tree in $V^{\mathbb{P}_{\delta}}$, with $N$ a transitive elementary substructure of $H\left(\delta^{+}\right)$of size $\delta$ containing $\mathbb{P}_{\delta}$ and $\dot{T}$ which is closed under $<\delta$ sequences and $j: N \rightarrow M$ an elementary embedding having critical point $\delta$. As before, we also assume that $|M|=\delta$ and $V \vDash " M^{<\delta} \subseteq M^{\prime}$ ". Write $j\left(\mathbb{P}_{\delta}\right)=\mathbb{P}_{\delta} * \dot{\mathbb{Q}}$. Regardless if $M \vDash " \delta$ is weakly compact", it will be the case that in $M, \Vdash_{\mathbb{P}_{\delta}}$ “Q் is $\prec \delta$-strategically closed". This means that the argument outlined in the paragraph immediately following the proof of Lemma 2.1 may now be 
used to show that $V^{\mathbb{P}_{\delta}} \vDash " \delta$ is weakly compact". This completes the proof of Lemma 2.6.

Lemma 2.7 In $V^{\mathbb{P}}, \delta$ is Mahlo and reflects stationary sets iff $\delta$ is weakly compact.

Proof: Suppose $V^{\mathbb{P}} \vDash " \delta$ is Mahlo and reflects stationary sets". Since by Lemmas 2.2 and 2.3, $V^{\mathbb{P}} \vDash " \kappa$ is supercompact and no cardinal is supercompact up to a Mahlo cardinal", it is clearly true that $\delta \leq \kappa$. Consequently, as $V^{\mathbb{P}} \vDash$ " $\kappa$ is weakly compact", we may assume that $\delta<\kappa$. In addition, since forcing does not create new Mahlo cardinals, we have that $V \vDash$ " $\delta$ is Mahlo".

We consider now two cases.

Case 1: $V \vDash " \delta$ is not weakly compact". Write $\mathbb{P}=\mathbb{P}_{\delta+1} * \dot{\mathbb{Q}}$. By the definition of $\mathbb{P}, \Vdash_{\mathbb{P}_{\delta+1}} " \delta$ contains a non-reflecting stationary set of ordinals of cofinality $\omega$ and forcing with $\dot{\mathbb{Q}}$ adds no new subsets of $\delta "$. Thus, $V^{\mathbb{P}_{\delta+1} * \dot{\mathbb{Q}}}=V^{\mathbb{P}} \vDash$ " $\delta$ does not reflect stationary sets".

Case 2: $V \vDash " \delta$ is weakly compact". Write $\mathbb{P}=\mathbb{P}_{\delta} * \dot{\mathbb{Q}}^{*}$. By Lemma 2.6, $V^{\mathbb{P}_{\delta}} \vDash$ " $\delta$ is weakly compact". Further, by the definition of $\mathbb{P}, \vdash_{\mathbb{P}_{\delta}}$ "Forcing with $\dot{\mathbb{Q}}^{*}$ adds no new subsets of $\delta$ ". Thus, $V^{\mathbb{P}_{\delta} * \dot{\mathbb{Q}}^{*}}=V^{\mathbb{P}} \vDash " \delta$ is weakly compact".

Since $V^{\mathbb{P}} \vDash$ " $\delta$ reflects stationary sets", $\delta$ cannot fall into the rubric of Case 1 . This means that $V \vDash " \delta$ is weakly compact", so by the proof given in Case $2, V^{\mathbb{P}} \vDash$ " $\delta$ is weakly compact" as well. Since weakly compact cardinals are both Mahlo and reflect stationary sets, this completes the proof of Lemma 2.7.

Lemmas $2.2-2.7$ and the intervening remarks complete the proof of Theorem 2 .

Theorem 3 now follows as an immediate corollary to Theorem 2 , with the model $V^{\mathbb{P}}$ constructed for Theorem 2 as its witness. To see this, it suffices to show that no strong cardinal $\delta<\kappa$ has its strongness indestructible under $\delta$-strategically closed forcing. However, if this were the case, then 
by Theorem 1, there would be non-weakly compact Mahlo cardinals $\gamma<\delta$ which reflect stationary sets. By the construction of $V^{\mathbb{P}}$, this is impossible. This completes the proof of Theorem 3.

There are some interesting questions left open by the above construction, with which we conclude this paper. They are as follows:

1. Is it possible to improve the levels of indestructibility for $\kappa$ to the levels given in [5] and [11], i.e., so that $\kappa$ 's supercompactness is indestructible under arbitrary $\kappa$-directed closed forcing and $\kappa$ 's strongness is indestructible under what Gitik and Shelah in [5] call " $\kappa^{+}$-weakly closed forcings satisfying the Prikry condition"?

2. Is it possible to prove an analogue of Theorem 2 in which there are Mahlo cardinals above $\kappa$ ?

3. Is it possible to prove an analogue of Theorem 3 for a universe with no restrictions on its large cardinal structure?

4. Is it possible to prove an analogue of Theorem 1 in which $\kappa$ is supercompact and exhibits some form of indestructibility for its supercompactness?

Observe that a positive answer to Question 3 would require as its witnessing model something quite different from the model for Theorem 2. Also, one way of obtaing a positive answer to Question 4 would be to construct a partial ordering $\mathbb{Q}^{*}$ analogous to the partial ordering $\mathbb{Q}$ of Lemma 2.1 (which may not even be $\aleph_{2}$-directed closed), but with a sufficient degree of directed closure.

\section{References}

[1] A. Apter, "Indestructibility and Level by Level Equivalence and Inequivalence between Strong Compactness and Supercompactness", Mathematical Logic Quarterly 53, 2007, 78-85.

[2] A. Apter, "Indestructibility and Measurable Cardinals with Few and Many Measures", submitted for publication to the Archive for Mathematical Logic. 
[3] A. Apter, J. Cummings, "Identity Crises and Strong Compactness II: Strong Cardinals", Archive for Mathematical Logic 40, 2001, 25-38.

[4] A. Apter, J. D. Hamkins, "Indestructibility and the Level-by-Level Agreement between Strong Compactness and Supercompactness", Journal of Symbolic Logic 67, 2002, 820-840.

[5] M. Gitk, S. Shelah, "On Certain Indestructibility of Strong Cardinals and a Question of Hajnal", Archive for Mathematical Logic 28, 1989, 35-42.

[6] J. D. Hamkins, "Gap Forcing", Israel Journal of Mathematics 125, 2001, 237-252.

[7] J. D. Hamkins, "Gap Forcing: Generalizing the Lévy-Solovay Theorem", Bulletin of Symbolic Logic 5, 1999, 264-272.

[8] J. D. Hamkins, "The Lottery Preparation", Annals of Pure and Applied Logic 101, 2000, $103-146$.

[9] K. Kunen, "Saturated Ideals", Journal of Symbolic Logic 43, 1978, 65-76.

[10] K. Kunen, Set Theory: An Introduction to Independence Proofs, Studies in Logic and the Foundations of Mathematics 102, North-Holland, Amsterdam and New York, 1980.

[11] R. Laver, "Making the Supercompactness of $\kappa$ Indestructible under $\kappa$-Directed Closed Forcing", Israel Journal of Mathematics 29, 1978, 385-388.

[12] R. Solovay, W. Reinhardt, A. Kanamori, "Strong Axioms of Infinity and Elementary Embeddings", Annals of Mathematical Logic 13, 1978, 73-116. 